\title{
Biopsy Site Re-localisation Based on the Computation of Epipolar Lines from Two Previous Endoscopic Images
}

\author{
Baptiste Allain ${ }^{1}$, Mingxing $\mathrm{Hu}^{1}$, Laurence B. Lovat ${ }^{2}$, Richard Cook ${ }^{3}$, \\ Sebastien Ourselin ${ }^{1}$, and David Hawkes ${ }^{1}$ \\ ${ }^{1}$ Centre for Medical Image Computing, University College London UCL \\ b.allain@ucl.ac.uk \\ ${ }^{2}$ Department of Gastroenterology, University College London Hospitals UCLH NHS \\ Foundation Trust and National Medical Laser Centre, Division of Surgical Sciences, UCL \\ ${ }^{3}$ Dental Institute, Department of Biomaterials, King's College London, KCL
}

\begin{abstract}
Tracking biopsy sites in endoscopic images can be useful to provide a visual aid for the guidance of surgical tools, for example when endoscopic guided biopsy is required. A new method for re-localisation of these sites is presented in this paper. It makes use of epipolar geometry properties between three images of the same site observed from different viewpoints with an endoscope. Two epipolar lines are derived from the two first images in the third image where the site needs to be re-localised. Their intersection corresponds to the location of the biopsy site. This method was tested with gastroscopic data from 2 patients with 9 series of three images of the oesophagus. The re-localisation error was estimated at less than 1.5 millimetres by a clinical endoscopist, which is sufficient for most clinical endoscopic applications.
\end{abstract}

\section{Introduction}

Endoscopy is a procedure for real-time video acquisitions of the interior of hollow organs using flexible or rigid instruments and facilitates taking biopsies. The main problem for many endoscopists is the detection of macroscopically invisible biopsy sites and re-localisation during the same or future examinations. CT and electromagnetic based systems for tracking the location of bronchoscopes have been described. These allow accurate targeting of biopsies in the relatively rigid bronchial tree $[1,2]$. A precision of re-localisation of $1.58 \mathrm{~mm}$ has been reported. Some examinations like gastroscopy are based, however, entirely on visual images. In Barrett's oesophagus, precancerous lesions may not be visible under white light endoscopy but may be detected histologically. It is important to be able to re-localise these lesions, particularly after taking a virtual 'optical' biopsy or in vivo histology based solely on the information within the endoscopy view. For several 'optical' biopsy techniques, one solution is to pass a miniprobe via the endoscope working channel and place it in contact with the tissue to scan the surface. The scan helps decide whether a tissue specimen needs to be taken. The forceps then need to be re-localised where the miniprobe was positioned. Unfortunately, the optical measurement and the excised tissue may not match, which can make the biopsy irrelevant if negative [3, 4]. Thus, the detected biopsy site may need to be re-localised precisely in subsequent video images. Methods for object 
localisation in video images have been recently addressed in the field of robotassisted surgery $[5,6]$, but have focused only on tracking the instrument tip. The scope of the biopsy site re-localisation focuses more on the projection of a point location from an image to another and requires the computation of the spatial transformations (rotations and translations) between endoscopic images. It has been demonstrated in [7] that the recovery of the epipolar geometry [8] between endoscopic images can be used to compute these spatial transformations.

The method presented in this paper proposes a solution to the re-localisation problem using robust feature tracking and the constraints of epipolar geometry for applications in gastroenterology. A point's location is computed in the target image as the intersection of two epipolar lines derived from the location of corresponding biopsy sites in pairs of previously acquired endoscopic images. The epipolar geometry is recovered with a robust technique. This method presents several advantages. (i) First, it reduces the computational burden since it is based only on the epipolar lines consideration. There is no need to directly compute the rotations and the translations between the images. (ii) Secondly, this method has the potential to be a real-time guidance system based on a limited amount of information. Biospy sites are tracked directly in the video images and not in relation to a pre-operative image. (iii) Finally, this method could be extended to any type of intra-operative optical imaging.

\section{Method}

\subsection{Re-localisation Principle}

If a biopsy site location is known in an endoscopic image $\mathbf{I}_{\mathbf{1}}$, it can be projected onto another image $\mathbf{I}_{2}$ for its re-localisation. This projection can be determined with the epipolar geometry formed with the two images $\mathbf{I}_{1}$ and $\mathbf{I}_{2}$. The epipolar geometry between $\mathbf{I}_{\mathbf{1}}$ and $\mathbf{I}_{\mathbf{2}}$ can be described algebraically using the fundamental matrix $\mathbf{F}_{\mathbf{1 2}}$ as:

$$
\mathbf{F}_{12}=\left(\begin{array}{lll}
f_{1} & f_{2} & f_{3} \\
f_{4} & f_{5} & f_{6} \\
f_{7} & f_{8} & f_{9}
\end{array}\right)=\mathbf{K}_{2}^{-\mathrm{T}}\left[\mathbf{t}_{12}\right] \mathbf{R}_{12} \mathbf{K}_{1}^{-1}=\mathbf{K}_{2}^{-\mathrm{T}}\left[\begin{array}{lcr}
0 & -t_{3} & t_{2} \\
t_{3} & 0 & -t_{1} \\
-t_{2} & t_{1} & 0
\end{array}\right] \mathbf{R}_{12} \mathbf{K}_{1}^{-1} .
$$

$\mathbf{K}_{1}$ and $\mathbf{K}_{2}$ are the intrinsic matrices of the camera at first and second positions defined from the focal length, the position of the image centre, and the scaling from the 3Dspace to the image. Once $\mathbf{F}_{\mathbf{1 2}}, \mathbf{K}_{\mathbf{1}}$ and $\mathbf{K}_{\mathbf{2}}$ are known, it is possible to determine the camera motions, rotation $\mathbf{R}_{\mathbf{1 2}}$ and translation $\mathbf{t}_{\mathbf{1 2}}$, with further computations.

During endoscopic procedures, a biopsy site can be seen from various viewpoints with an endoscopic camera (Fig. 1). Let $\mathbf{I}_{\mathbf{1}}$ and $\mathbf{I}_{\mathbf{2}}$ be two images where the biopsy site location is visible and $\mathbf{I}_{\mathbf{3}}$ be a third image where it needs to be re-localised. Let $\mathbf{P}$ be the biopsy site location in the 3D space, and $\mathbf{p}_{\mathrm{T} 1}$ and $\mathbf{p}_{\mathrm{T} 2}$ be the locations of the biopsy site in images $\mathbf{I}_{1}$ and $\mathbf{I}_{2}$. The fundamental matrices $\mathbf{F}_{13}$ and $\mathbf{F}_{23}$ can be computed between respectively images $\mathbf{I}_{\mathbf{1}}$ and $\mathbf{I}_{\mathbf{3}}$ and images $\mathbf{I}_{\mathbf{2}}$ and $\mathbf{I}_{\mathbf{3}}$. The axes formed with camera centre 1 and camera centre 3 , and camera centre 2 and camera centre 3 , have an intersection with the image plane $\mathbf{I}_{\mathbf{3}}$, which is called the epipole. Let $\mathbf{e}^{\mathbf{1 3}}$ and $\mathbf{e}^{\mathbf{2 3}}$ be the two epipoles of this configuration. Thus, $\mathbf{F}_{13} \mathbf{p}_{\mathbf{T} 1}$ is a vector and defines the epipolar line $\boldsymbol{l}_{\boldsymbol{l}}$, which passes through the projection of $\mathbf{p}_{\mathrm{T} 1}$ onto $\mathbf{I}_{\mathbf{3}}$ and through $\mathbf{e}^{\mathbf{1 3}}$. The 


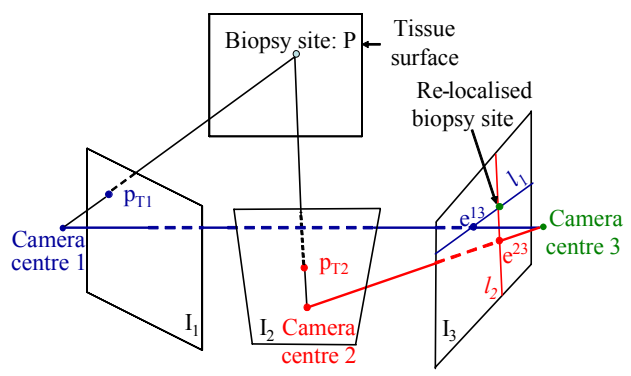

Fig. 1. Method for biopsy site re-localisation with three images and two epipolar lines

epipolar line $\boldsymbol{l}_{\mathbf{2}}$ can be defined similarly for $\mathbf{F}_{\mathbf{2 3}} \mathbf{p}_{\mathbf{T} \mathbf{2}}$. As $\mathbf{p}_{\mathbf{T} \mathbf{1}}$ and $\mathbf{p}_{\mathbf{T} \mathbf{2}}$ correspond to the same biopsy site location in the 3D-space, the intersection of $\boldsymbol{l}_{\boldsymbol{l}}$ and $\boldsymbol{l}_{\boldsymbol{2}}$ returns the location of the biopsy site in image $\mathbf{I}_{\mathbf{3}}$.

\subsection{Re-localisation System}

The two epipolar lines are determined with computations described in [8]. As summarised in Fig. 2, two pairs of endoscopic images are provided as inputs to the system and processed independently until step 5.

The system is described as follows for Input 1:

\section{Feature Tracking and Matching}

As described in [9] for bronchoscopy, a block matching in step 1 tracks blocks of $M \times N$ pixels through a series of subsequent images to match features $\left\{\mathbf{p}_{1 \mathrm{i}}, \mathbf{p}_{3 \mathrm{i}}\right\}$ from images $\mathbf{I}_{\mathbf{1}}$ and $\mathbf{I}_{\mathbf{3}}$. The similarity between two blocks, $\mathbf{B} \mathbf{m}_{i}$ and $\mathbf{B n}_{i}$, in images $\mathrm{m}$ and $\mathrm{n}$ is the square of the cross-correlation of each block with pixel intensities $\mathbf{B m}_{i}(k, l)$ and $\mathbf{B n}_{i}(k, l)$, average intensities $\mathbf{B m}_{i 0}$ and $\mathbf{B} \mathbf{n}_{i 0}$, and standard deviations $\sigma_{B m i}$ and $\sigma_{B n i}$ :

$$
C C^{2}\left(\mathbf{B} \mathbf{m}_{i}, \mathbf{B} \mathbf{n}_{i}\right)=\frac{\left[\sum_{k=1}^{M} \sum_{l=1}^{N}\left(\mathbf{B} \mathbf{m}_{i}(k, l)-\mathbf{B} \mathbf{m}_{i 0}\right)\left(\mathbf{B} \mathbf{n}_{i}(k, l)-\mathbf{B} \mathbf{n}_{i 0}\right)\right]^{2}}{\sigma_{B m_{i}}^{2} \sigma_{B n_{i}}^{2}} .
$$

\section{Inliers' Detection}

The Maximum A Posteriori Sample Consensus (MAPSAC) has been presented as a robust method for inliers' detection among the features [10]. The error for matched features must be minimised, which is equivalent to minimising a cost function $C$ :

$$
C=\sum_{i=1 . . n} \rho\left(e_{i}^{2}\right) \text { with } \rho\left(e_{i}^{2}\right)=\left\{\begin{array}{cc}
e_{i}^{2} & \text { if } e_{i}^{2}<T \\
T & \text { if } e_{i}^{2} \geq T
\end{array}\right\} .
$$

As explained in [10], $T$ is a threshold for the detection of inliers and $e_{i}$ is the geometric distance for matched features $\left\{\mathbf{p}_{1 \mathrm{i}}, \mathbf{p}_{3 \mathrm{i}}\right\}$. The contribution of the inliers to the error and to the fundamental matrix computation is, therefore, taken into account. 


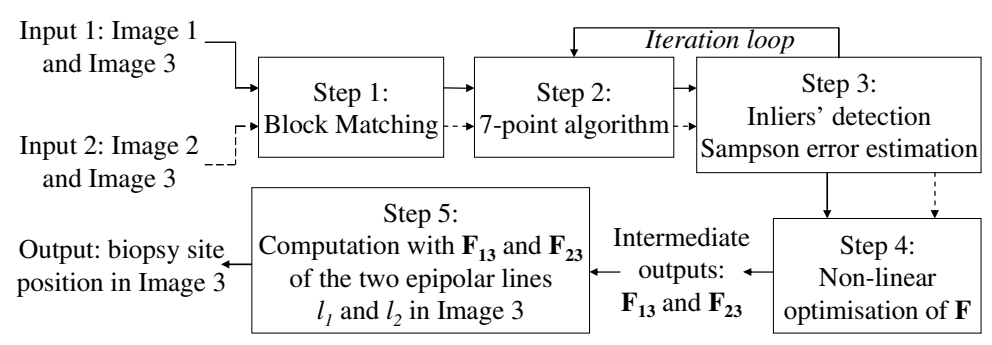

Fig. 2. Block diagram of the biopsy site re-localisation algorithm

\section{Determination of the Epipolar Lines Intersection}

Steps 2 and 3 are iteratively run over samples of 7 matched features. For each sample, the 7-point algorithm is applied [8] and returns one or three solutions for the fundamental matrix $\mathbf{F}_{\mathbf{1 3}}$. The cost function $C$ is applied with the Sampson distance $e_{i}$ :

$$
e_{\mathrm{i}}^{2}=\frac{\left(\mathbf{p}_{3 \mathrm{i}}^{\mathrm{T}} \mathbf{F}_{13} \mathbf{p}_{1 \mathrm{i}}\right)^{2}}{\left(\mathbf{F}_{13} \mathbf{p}_{1 \mathrm{i}}\right)_{1}^{2}+\left(\mathbf{F}_{13} \mathbf{p}_{1 \mathrm{i}}\right)_{2}^{2}+\left(\mathbf{F}_{13}{ }^{\mathrm{T}} \mathbf{p}_{3 \mathrm{i}}\right)_{1}^{2}+\left(\mathbf{F}_{13}{ }^{\mathrm{T}} \mathbf{p}_{3 \mathrm{i}}\right)_{2}^{2}} .
$$

$\left(\mathbf{F}_{\mathbf{1 3}} \mathbf{p}_{1 \mathrm{i}}\right)_{1}$ is the $1^{\text {st }}$ component of the vector $\mathbf{F}_{\mathbf{1 3}} \mathbf{p}_{1 \mathrm{i}}$. This error expresses how well $\mathbf{F}_{\mathbf{1 3}}$ fits the matched features $\left\{\mathbf{p}_{1 \mathrm{i}}, \mathbf{p}_{3 \mathrm{i}}\right\}$. After the iteration loop, $\mathbf{F}_{\mathbf{1 3}}$ minimizes $C$. A second estimation of $\mathbf{F}_{\mathbf{1 3}}$, minimising the Sampson distance, is found in step 4 with a constrained non-linear optimisation applied to the inliers detected from the MAPSAC. Thus, in step 5, the required epipolar lines $l_{1}$ and $l_{2}$ for the biopsy site re-localisation are computed from $\mathbf{F}_{13}$ and $\mathbf{F}_{23}$ and return the expected result.

\section{Validation}

\subsection{Materials and Method}

Purpose. The validation consisted of first checking the recovery of the epipolar geometry to make sure that reliable epipolar lines could be computed and secondly of assessing the re-localisation error.

Data and Procedure. In vivo data were acquired with monocular endoscopes during gastroscopy of two patients. The same endoscopist introduced a $2 \mathrm{~mm}$ diameter miniprobe into the working channel of the endoscope, scanned the tissue surface, moved the endoscope camera to change the viewpoints, and removed the miniprobe. The relocalisation method was validated with 9 groups of 3 images from the 2 patients. Cardiac and breathing motion could be approximated by an affine transformation of the oesophagus surface.

Validation of the Recovery of the Epipolar Geometry. Endoscope motion usually comprises small rotations and translations around and along the optical axis of the endoscope in consecutive images. Thus, every time the re-localisation algorithm was applied, the computed epipoles were visually checked to ensure that they were in an 


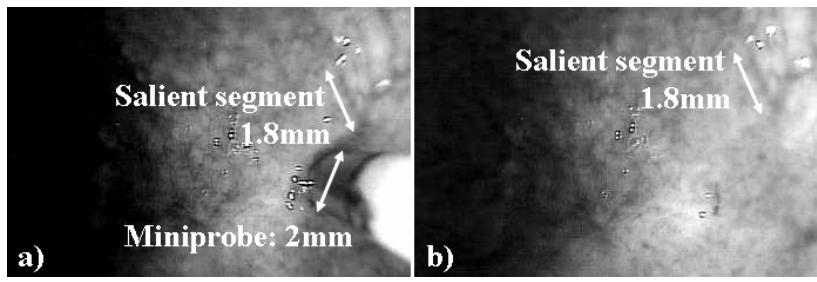

Fig. 3. Scales in the endoscopic images: a) The miniprobe helps compute the size of the vessel, b) which was used as a scale in Image 3

area in reasonable agreement with the endoscope motions. The total Sampson distance, defined as:

$$
d=\sqrt{\frac{1}{n} \cdot \sum_{i=1}^{n} \frac{\left(\mathbf{p}_{3 \mathrm{i}}^{\mathrm{T}} \mathbf{F}_{13} \mathbf{p}_{1 \mathrm{i}}\right)^{2}}{\left(\mathbf{F}_{13} \mathbf{p}_{1 \mathrm{i}}\right)_{1}^{2}+\left(\mathbf{F}_{13} \mathbf{p}_{1 \mathrm{i}}\right)_{2}^{2}+\left(\mathbf{F}_{13}{ }^{\mathrm{T}} \mathbf{p}_{3 \mathrm{i}}\right)_{1}^{2}+\left(\mathbf{F}_{13}{ }^{\mathrm{T}} \mathbf{p}_{3 \mathrm{i}}\right)_{2}^{2}}} .
$$

was computed for the fundamental matrices $\mathbf{F}_{\mathbf{1 3}}$ and $\mathbf{F}_{\mathbf{2 3}}$, obtained with: (1) the Normalised 8-Point (N8P) algorithm [8] applied to all the feature matches $\left\{\mathbf{p}_{1 \mathrm{i}}, \mathbf{p}_{3 \mathrm{i}}\right\}$ or $\left\{\mathbf{p}_{2 \mathrm{i}}, \mathbf{p}_{3 \mathrm{i}}\right\}$, and (2) with the re-localisation algorithm.

Estimation of the Re-localisation Error. This was carried out by the endoscopist. A biopsy site (a point on a salient vessel at the tissue surface or the miniprobe tip) was manually selected in Images 1 and 2. It was re-localised both visually in Image 3 to return a ground-truth location and automatically with the re-localisation algorithm. An error in pixels and in millimetres was determined from these two locations. As the diameter of the miniprobe was $2 \mathrm{~mm}$, the size of a salient segment, e.g. a vessel, in the same plane as the miniprobe could be computed (Fig. 3 a)). This salient segment defined a scale in Image 3 for the conversion of the error from pixels to millimetres and for an estimation of the tissue dimensions in the Field Of View (FOV) of the image (Fig. 3 b)).

\subsection{Results}

Recovery of the Epipolar Geometry. As indicated in Fig. 4, the epipoles $\mathbf{e}^{\mathbf{1 3}}$ and $\mathbf{e}^{\mathbf{2 3}}$ computed for the pairs of images 1 and 3 (I 1-3), and 2 and 3 (I 2-3) were in an area in agreement with the endoscope motions. The re-localisation algorithm gave an order of magnitude reduction in the Sampson distance in comparison to the N8p algorithm. Thus, reliable epipolar geometry was recovered with the re-localisation algorithm.

Re-localisation: The estimated errors in pixels and in millimetres for the nine groups of three endoscopic images are presented in Table 1. This error varied from 2 pixels to 50 pixels in the $\mathrm{x}$ and $\mathrm{y}$ directions of the image. The endoscopist assessed the error for each group of images in millimetres with the scale that was provided in Image 3 . In most cases, this error was smaller than $1.5 \mathrm{~mm}$ which corresponds to approximately 100-150 cells. In practice, an extracted tissue sample has a diameter of $5 \mathrm{~mm}$; therefore, the endoscopist would have a high chance of extracting a part of the region that he previously analysed with the miniprobe using forceps. The errors greater than $1.5 \mathrm{~mm}$ 


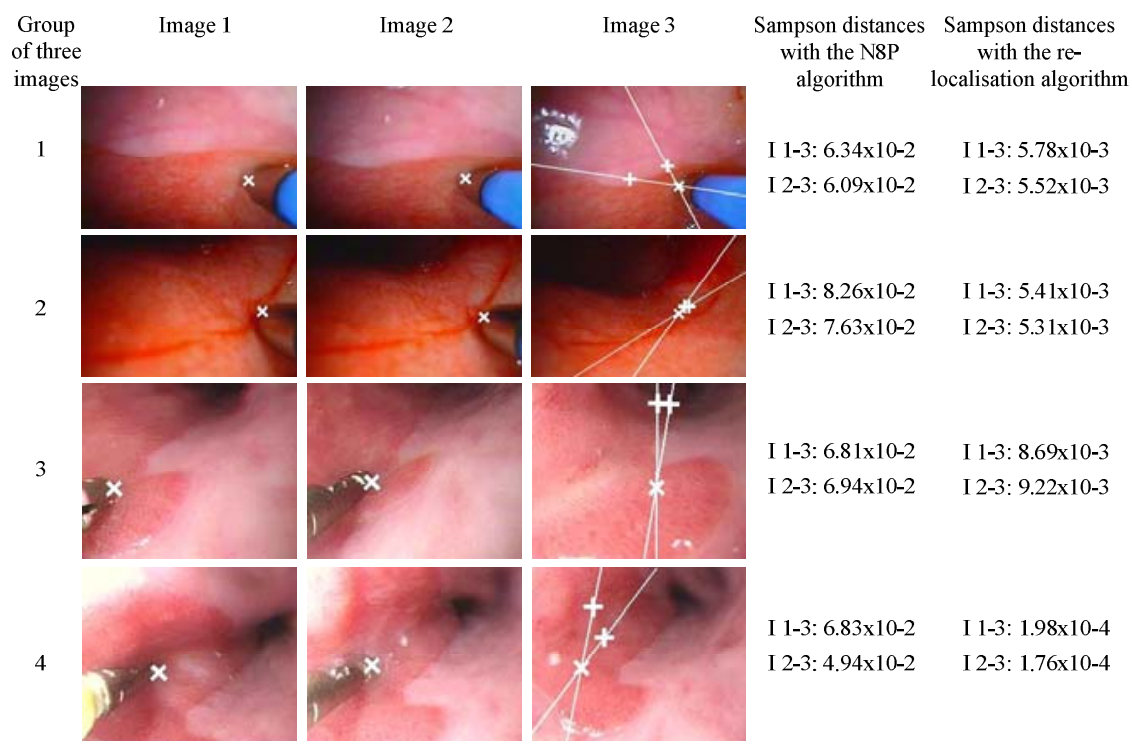

Fig. 4. Results for the recovery of the epipolar geometry and for the re-localisation of a biopsy site: the computed epipoles $\mathbf{e}^{\mathbf{1 3}}$ and $\mathbf{e}^{\mathbf{2 3}}$ are described with the sign ' + ' in Image 3 for each group of images. The sign ' $\mathrm{x}$ ' describes the biopsy site, whose positions $\mathbf{p}_{\mathbf{T} 1}$ and $\mathbf{p}_{\mathbf{T} 2}$ are manually selected in Image 1 and Image 2 . In Image 3, the sign ' $x$ ' describes the biopsy site computed with the two epipolar lines shown in Image 3. Two Sampson distances are computed in pixels for each group of images: one for the pair of images I 1-3, and the other for the pair I 2-3.

Table 1. Re-localisation errors in pixels and estimation of the absolute errors in millimeters

\begin{tabular}{ccccc}
\hline Image & $\begin{array}{c}\text { Dimension } \\
\text { (pixels) }\end{array}$ & $\begin{array}{c}\text { FOV } \\
(\mathbf{m m})\end{array}$ & $\begin{array}{c}\text { Error } \\
\text { (pixels) }\end{array}$ & $\begin{array}{c}\text { Error } \\
\text { (mm) }\end{array}$ \\
\hline $\mathbf{1}$ & $229 \times 344$ & $20 \times 15$ & $1.3 \times 7.6$ & 0.60 \\
$\mathbf{2}$ & $229 \times 344$ & $30 \times 30$ & $12.8 \times 24.7$ & 1.39 \\
$\mathbf{3}$ & $229 \times 344$ & $30 \times 30$ & $1.9 \times 0.5$ & 0.52 \\
$\mathbf{4}$ & $229 \times 344$ & $30 \times 30$ & $14.9 \times 26$ & 0.59 \\
$\mathbf{5}$ & $229 \times 344$ & $15 \times 10$ & $47.9 \times 12.4$ & 1.28 \\
$\mathbf{6}$ & $193 \times 235$ & $15 \times 20$ & $29.2 \times 3.4$ & 1.40 \\
$\mathbf{7}$ & $193 \times 235$ & $30 \times 30$ & $12.9 \times 3.6$ & 1.22 \\
$\mathbf{8}$ & $193 \times 235$ & $30 \times 30$ & $2.2 \times 32.5$ & 2.56 \\
$\mathbf{9}$ & $193 \times 235$ & $20 \times 30$ & $8.3 \times 31.2$ & 2.13 \\
\hline
\end{tabular}

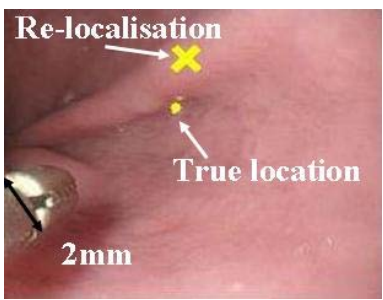

Fig. 5. Re-localisation error

become much more critical. They usually occur in endoscopic images with a poor contrast. In Fig. 5 for example, the tissue texture was smooth: no vessel or other salient structure existed in this region. Therefore, the main features extracted with the block matching were localised in the tissue folds, or in zones with a weak contrast. The features were sensitive to the endoscope motions and to the changes in illumination. Several of them were inaccurately tracked by a few pixels but were not detected as outliers since they did not create large errors. Thus, for these cases, the recovery of 
the epipolar geometry was inaccurate and the epipolar lines derived from the biopsy site in Image 1 and 2 did not pass through the actual location of the biopsy site in Image 3 .

\section{Conclusion}

A method for the re-localisation of biopsy sites in endoscopic images has been described. It has the advantages of using epipolar geometry properties without directly computing the rotations and the translations of the endoscope camera. This method needs only the determination of the fundamental matrix to compute the necessary epipolar lines. Clinical data validation showed re-localisation can be accurate to less than $1.5 \mathrm{~mm}$ or to within $100-150$ cells as determined by the direct scaling of images and independent corroboration by an endoscopist. Although tested only with gastroscopic images, this method should work equally well for other endoscopic applications. Regions with greater structure and contrast would improve the ease and accuracy of the final re-localisation results. The technique is robust as satisfactory results were obtained even with substantial camera motions between video images.

Future work includes refining the re-localisation error by improving (a) both the feature tracking and matching, and (b) outlier detection. The re-localisation algorithm may have greater difficulty in cases where the endoscope returns to the biopsy site region at a later time during the examination or subsequent investigations. Thus, future work will also focus on (c) an extension of the method to the cases when a biopsy site needs to be re-localised with independent video sequences and not only successive video images from a single sequence. Finally, (d) a phantom study is required to estimate the re-localisation errors more accurately.

Acknowledgments. This work was undertaken at UCLH/UCL with funding from the Department of Health (UK) DLH Clinician Scientist Award to Dr. Cook, Engineering and Physical Sciences Research Council (EPSRC - grant reference EP/C523016/1), Department of Health's NIHR Comprehensive Biomedical Research Centre (UCLH), Cancer Research UK: grant to the UCL Experimental Cancer Medicine Centre. The views expressed in this publication are those of authors and not necessarily those of the funders.

\section{References}

1. Helferty, J.P., Sherbondy, A.J., Kiraly, A.P., et al.: Computer-based system for the virtualendoscopic guidance of bronchoscopy. Computer Vision and Image Understanding 108(12), 171-187 (2007)

2. Mori, K., Deguchi, D., Ishitani, K., Kitasaka, T., Suenaga, Y., Hasegawa, Y., Imaizumi, K., Takabatake, H.: Bronchoscope tracking without fiducial markers using ultra-tiny electromagnetic tracking system and its evaluation in different environments. In: Ayache, N., Ourselin, S., Maeder, A. (eds.) MICCAI 2007, Part II. LNCS, vol. 4792, pp. 644-651. Springer, Heidelberg (2007) 
3. Lovat, L.B., Johnson, K., Mackenzie, G.D., et al.: Elastic scattering spectroscopy accurately detects high grade dysplasia and cancer in Barrett's oesophagus. Gut. 55, 1078-1083 (2006)

4. Pohl, H., et al.: Miniprobe confocal laser microscopy for the detection of invisible neoplasia in patients with Barrett's oesophagus. Gut 57, 1648-1653 (2008)

5. Speidel, S., Delles, M., Gutt, C., et al.: Tracking of Instruments in Minimally Invasive Surgery for Surgical Skill Analysis. In: Yang, G.-Z., Jiang, T.-Z., Shen, D., Gu, L., Yang, J. (eds.) MIAR 2006. LNCS, vol. 4091, pp. 148-155. Springer, Heidelberg (2006)

6. Voros, S., Long, J.A., Cinquin, P.: Automatic Localization of Laparoscopic Instruments for the Visual Servoing of an Endoscopic Camera Holder. In: Larsen, R., Nielsen, M., Sporring, J. (eds.) MICCAI 2006. LNCS, vol. 4190, pp. 535-542. Springer, Heidelberg (2006)

7. Hu, M., Penney, G., Edwards, P., et al.: 3D reconstruction of internal organ surfaces for minimal invasive surgery. In: Ayache, N., Ourselin, S., Maeder, A. (eds.) MICCAI 2007, Part I. LNCS, vol. 4791, pp. 68-77. Springer, Heidelberg (2007)

8. Hartley, R., Zisserman, A.: Multiple View Geometry in Computer Vision. Cambridge University Press, Cambridge (2004)

9. Mori, K., Deguchi, D., Sugiyama, J., et al.: Tracking of a bronchoscope using epipolar geometry analysis and intensity-based image registration of real and virtual endoscopic images. Medical Image Analysis 6(3), 321-336 (2002)

10. Torr, P.H.S., Davidson, C.: IMPSAC: A synthesis of importance sampling and random sample consensus. IEEE Transactions on Pattern Analysis and Machine Intelligence 25(3), 354-364 (2003) 